\title{
Conrad Gesner et la Zoologie: les Invertébrés
}

\author{
Par Jean Théodoridès
}

Il y a exactement quatre siècles aujourd'hui que s'éteignait ici même à Zürich, le 13 décembre 1565, Conrad Gesner, victime d'une épidémie de peste qu'il essayait de combattre en tant que médecin de la ville.

Mais Gesner fut également un très grand naturaliste qui publia une gigantesque encyclopédie zoologique intitulée Historia animalium totalisant 4500 pages in folio.

Nous n'insisterons pas ici sur la biographie de Gesner et son apport concernant les Vertébrés, ces aspects de sa vie et de son œuvre ayant été évoqués avec une très grande compétence par notre maître et ami le Professeur G. Petit dans la belle conférence qu'il fit à Genève le 24 septembre $1965^{1}$.

Il faut cependant rappeler que quatre volumes consacrés aux Quadrupèdes vivipares et ovipares, aux Oiseaux et aux animaux aquatiques parurent du vivant de Gesner, de 1551 à 1558, et furent complétés en 1560, par des atlas de planches (Icones animalium quadrupedum..., Icones avium omnium, Nomenclator aquatilium animantium).

Après sa mort, fut publié un cinquième volume consacré aux serpents (De serpentibus) auquel il est joint une courte monographie du scorpion (De scorpione) (1587).

Gesner avait réuni des matériaux en vue d'un sixième volume concernant les insectes; nous verrons plus loin quelles furent les vicissitudes de cet ouvrage.

Dans le cadre limité de cet exposé, nous voudrions surtout examiner la contribution de Gesner à l'étude des Invertébrés qui a été jusqu'ici relativement peu étudiée.

Ceux-ci sont traités dans le volume sur les animaux aquatiques (... de piscium et aquatilium animantium natura, Froschover, Zürich 1558, 1297 pages in folio) et dans le Nomenclator aquatilium animantium (même éditeur, 1560), dans le De scorpione cité ci-dessus et enfin dans l'ouvrage posthume de Moufet sur les insectes (1634).

1 G. Petit, Conrad Gesner, zoologiste, Gesnerus 22 (1965) 195-204. 
Avant de considérer cet apport de Gesner à la connaissance des animaux inférieurs, il convient de rappeler la méthode qu'il emploie dans son encyclopédie où on retrouve les préoccupations littéraires et linguistiques si caractéristiques de son esprit et de son époque. Il établit en effet huit sections ou chapitres, désignés par les huit premières lettres de l'alphabet et se répétant pour chacun des animaux décrits: nom de l'animal dans les différentes langues anciennes et modernes, son origine, son habitat et ses caractères morphologiques externes et internes, ses «fonctions naturelles» (sorte de physiologie) et ses maladies, mœurs, instincts, psychisme et utilité générale de l'animal, son rôle alimentaire, son rôle en médecine, enfin spéculation poétiques et philosophiques, anecdotes et proverbes.

Ajoutons que comme les compilateurs médiévaux, Gesner classe encore les espèces étudiées par ordre alphabétique. Comme l'a bien dit Emile Guyéмот ${ }^{2}$ : “C'est, en somme, un dictionnaire zoologique.» Cependant, dans ses Icones... et dans son Nomenclator..., Gesner présente les animaux en les répartissant par ordres et donne pour chaque espèce: une courte diagnose en quelques lignes, les noms de l'animal dans diverses langues, une ou plusieurs figures.

C'est dans ce dernier ouvrage (p. 85-274) que Gesner considère in fine cinq ordres d'Invertébrés marins correspondant aux Anaima d'ARIstote et intitulés: De Mollibus, De Crustatis, De Testaceis, De Insectis marinis, De Zoophytis marinis.

Voyons quelle en est la composition.

Le premier ordre (De Mollibus) comprend les Mollusques Céphalopodes (Sepia, Sepiola, Loligo, Octopus) auxquels Gesner ajoute sous le nom de Nautilus, l'Argonaute (Argonauta argo) que Pierre Belon (1555) plaçait dans les Testacés alors que Rondelet (1558) le rattachait déjà aux autres Céphalopodes.

Gesner classe encore dans le même ordre des Mollusques Gastéropodes comme l'Aplysie, des Coelentérés (Actinies avec bonne représentation des aconties p. 197, diverses méduses) et des Crustacés (balanes).

Le second ordre (De Crustatis) comprend de véritables Crustacés Décapodes Brachyoures ${ }^{3}$ : Portunus (= Macropipus), Pisa, Calappa, Maia,

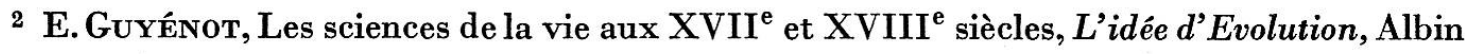
Michel, Paris 1941, p. 48.

3 Pour les crabes cités par Gesner, voir M.D. Grmek et D. Gurnot, Les crabes chez Ulysse

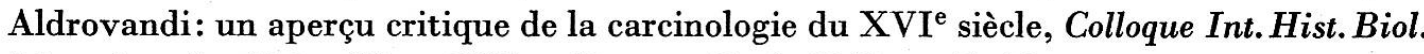
Mar., Suppl. ${ }^{\circ} 19$ à Vie et Milieu, Masson, Paris 1965, p. 45-64. 
Macropodia, Pinnoteres; Anomoures (pagures dont l'un p. 209 a l'aspect d'un scorpion!) et Macroures: Gammarus (= Homarus), Palinurus (langouste), Crangon et diverses Crevettes.

Sous le nom de Squilla, Gesner décrit la «cigale de mer» (Scyllarus arctus) et sous celui de Mantis locusta, la squille (Squilla mantis) qui est un Crustacé Stomatopode.

Dans le troisième ordre (De Testaceis) on trouve pêle-mêle des Mollusques Gastéropodes (Patella, Murex, Buccinum etc...) et Bivalves: Ostrea, Tapes, Pecten, Pinna etc..., des Brachiopodes et des Echinodermes (oursins, Etoiles de mer, Ophiures, Gorgonocephalus, holothuries) ce qui, comme l'a fait remarquer à juste titre $\mathrm{E}$. Guyénot, enlève de l'homogénéité à l'ensemble.

Le quatrième ordre (De insectis marinis) est le plus hétérogène. On y trouve en effet un poisson (l'hippocampe), des Annélides Polychètes décrites et figurées sous le nom de Scolopendra marina et Eruca marina (= Aphrodite aculeata). Sous le nom de Pediculus marinus, Gesner décrit et figure dans ce groupe un Isopode Cirolanide (Anilocra), sous celui d'Asilus marinus un autre Crustacé (Cymothoïde ?) tous deux parasites de Poissons.

On trouve aussi dans cet ordre des dentales et des sangsues.

Dans le cinquième ordre (De Zoophytis marinis) figurent des Bryozoaires, des Coelentérés (Vérétille, Alcyon) et des Tuniciers (colonie de Botrylles appelée par Gesner Uva marina).

Enfin, dans le dernier livre consacré aux animaux d'eau douce, Gesner cite et figure divers Invertébrés répartis en trois groupes: Crustacés (écrevisse), Testacés (divers Gastéropodes: limnée, Unio, anodonte, escargots, limaces) et Insectes (Hémiptères aquatiques: nèpe, notonecte; larves de phryganes et de divers Coléoptères; Vers: sangsue, gordien).

Dans le court traité sur le scorpion (De scorpione) déjà mentionné, Gesner décrit cet arthropode et ses mœurs mais croit à l'existence d'individus ailés (la figure représente plutôt des élytres) en se fiant à Pline, Strabon, Nicandre, Elien et Avicenne. Cette erreur sera partagée par d'autres naturalistes du $\mathrm{XV}^{\mathrm{e}}$ siècle (Jean Bauhin, Mattioli).

Si l'on examine la classification des Invertébrés qu'adopte Gesner, on réalise tout de suite que tributaire de l'Antiquité classique, il a purement et simplement suivi Aristote qui divisait les anaima en Mollusques, Malacostracés, Testacés, Entoma et reliquia.

Et c'est exactement la même subdivision qu'adopteront d'autres zoologistes du XVI ${ }^{\mathrm{e}}$ siècle tels que Wotton, Pierre Belon, Aldrovandi et du XVII ${ }^{\mathrm{e}}$ siècle (Jonston). 
Le regretté Paul Delaunay a donné dans un ouvrage posthume sur la Zoologie au $\mathrm{XVI}^{\mathrm{e}}$ siècle ${ }^{4}$ un tableau comparatif des classifications des Invertébrés adoptées par Aristote, Pline, Belon et Aldrovandi et celles-ci correspondant étroitement.

Ce n'est qu'au XVII ${ }^{e}$ siècle avec John RAY et surtout au XVIII ${ }^{e}$ avec LiNNÉ que la taxonomie des animaux inférieurs sera perfectionnée par la recherche et l'établissement de groupements naturels.

Un des mérites de Gesner est d'avoir pour la première fois réuni dans ses Testacés les principaux types d'Echinodermes (oursin, étoile de mer, ophiure, Gorgonocephalus, holothurie) mais, comme l'a souligné E. Guyénot ${ }^{5}$, c'est probablement par hasard.

Il nous faut maintenant considérer le curieux sort qu'eurent les notes et figures sur les insectes réunies par Gesner ${ }^{6}$.

Ces documents furent transmis par JoAChIm II CAmerarius (1534-1598) médecin et botaniste qui hérita de la bibliothèque botanique de Gesner léguée à Thomas Penn de Londres.

Ce dernier envisageait de les publier en y ajoutant des extraits de Wotton et travailla pendant quinze ans à ce volume que sa mort l'empêcha de terminer.

C'est un autre anglais, Thomas Moufet ${ }^{7}$ (ou Moffet) (1550-1600 ou 1553-1604) qui devait venir à bout de l'ouvrage qui fut publié après sa mort en 1634 sous le nom de Insectorum sive minimorum animalium theatrum par les soins du médecin de Charles $I^{\text {er }}$ qui répondait au nom curieux de Turquet de Mayerne (1573-1655).

Bien que nous ne sachions donc pas quelle est la part exacte qui revient à Gesner dans son élaboration, nous devons examiner rapidement le contenu de ce volume et sa valeur scientifique.

L'ouvrage de Moufet est divisé en 2 livres et en 42 chapitres.

Le premier livre (29 chapitres) concerne les insectes ailés (Hyménoptères, Diptères, Lépidoptères, Coléoptères, Orthoptéroïdes, Hémiptères). Les

4 Paul Delaunay, La Zoologie au XVI $I^{e}$ siècle, Hermann, Paris 1962.

5 E. GuYÉnOT, op.cit., 52.

6 Selon le Dr P.Delaunay, op.cit., p. 222 : « Gesner avait réuni pour la partie entomologique de son encyclopédie des notes et figures sur bois, aujourd'hui conservées à la bibliothèque de Zürich. » Le Professeur Ackerknecht a bien voulu nous préciser que ces documents ne s'y trouvent plus. Ils ne sont également pas à Erlangen où se trouvent de nombreux manuscrits de Gesner et il est probable qu'ils ont été envoyés en Angleterre où l'on a perdu leur trace.

7 W.J.Bishop, Dr. Thomas Muffett, a pioneer entomologist, Brit. Med.Bull. 3 (1945) 239. 
abeilles sont traitées en premier et Moufet croit encore à la légende selon laquelle elles naîtraient à partir du cadavre d'un taureau, la reine (qu'il prend pour un mâle) se formant dans le cerveau du Bovidé!

On retrouve au chapitre XXVIII la description et la figure du scorpion à élytres déjà publiée par Gesner.

Dans le second livre (42 chapitres) sont traités les insectes aptères. On y trouve des chenilles et des larves d'espèces ailées, des Crustacés (cloportes), Arachnides et Myriapodes, des ectoparasites (puces, poux, punaises), des Vers (Helminthes intestinaux, sangsue, Gordius).

L'iconographie est très inégale. Certaines gravures (Lépidoptères, Coléoptères, Orthoptéroïdes, Iule) sont très fidèles, d'autres fantaisistes (Méloïde du chapitre XXIII figuré avec un visage humain, scorpion à élytres, etc...).

A la fin du volume sont données 4 planches de figures où l'on trouve entre autres des Polychètes Sédentaires (hermelles?) et d'autres dessins (Physalus, Oestrus, Hippocampe) empruntés à l'Historia animalium de Gesner ainsi qu'une bonne représentation de scolopendre et une très médiocre d'un ténia.

Cuvier a dit de Moufet «qu'il était pour les insectes ce que Gesner est pour les quadrupèdes et Rondelet pour les poissons». Toujours selon lui: «son livre est le premier traité un peu complet... sur cette branche de la Zoologie. La division des insectes y est... encore assez imparfaite; son ouvrage n'est encore qu'un travail assez incomplet» mais remarquable par le nombre des figures (environ 500) «d'après nature et la plupart assez exactes».

Pour E. Guyénot (op.cit. p. 57), «l'ouvrage n'est ni meilleur ni pire que celui d'Aldrovandi. Il n'y a aucun effort d'observation personnelle ou de jugement. On retrouve les mêmes erreurs traditionnelles, les mêmes confusions, la même réunion des Insectes, Myriapodes, Arachnides et Vers, en un groupe parfaitement hétérogène.»

Nous ne pouvons que souscrire à ce dernier jugement. Bien que le microscope n'ait pas encore été au point à son époque, Moufet est difficilement excusable d'avoir méconnu des affinités zoologiques basées sur des caractères visibles à l'œil nu ou à la loupe et qu'Aristote avait parfaitement reconnues.

Comme l'écrit avec justesse CARus: «Il n'a rien vérifié, rien cherché à observer lui-même. Il eût suffi pour éviter certaines erreurs de quelques observations qui ne demandaient qu'un peu de soin.» 
Il convient maintenant de dire quelques mots de l'iconographie des Invertébrés illustrant l'œuvre de Gesner et en particulier des Icones animalium.

Ces dessins sont dus à des artistes dont les noms nous sont connus: les Zürichois Jean Asper et Jean Thomas, Lugas Schroen (figures d'oiseaux).

Ceux-ci sont des gravures sur bois (xylographies) «d'une suffisante exactitude, dessinées d'un trait louable quoique un peu mince et sec» (P. Delaunay).

Certaines de ces planches ont été empruntées aux ouvrages de Rondelet, Belon et Salviani, tandis que d'autres sont propres à l'ouvrage de Gesner et seront utilisées par Aldrovandi.

Ce sont de véritables illustrations passe-partout «échangées entre éditeurs, ou recopiées sans souci de la propriété artistique, et parfois avec méprises et confusions» (P. Delaunay).

Dans les Icones animalium, elles sont souvent moins parfaites que dans l'ouvrage complet (ceci ne semble pas être le cas pour les espèces aquatiques) et dans une lettre à son correspondant CAÏUs (Epist.Med. 1577, fol. 135) Gesner s'en plaint amèrement.

Nous noterons parmi les meilleures celles représentant la seiche (p. 186), les sépioles (p. 187); le calmar (p. 188), le poulpe (p. 190) les actinies (p. 197), le crabe tourteau (p. 202), la cigale de mer (p. 216), la squille (p. 220), les Gastéropodes marins (p.242-256), les oursins (p. 257); Gorgonocephalus (p. 261).

Parmi les plus médiocres: certains pagures (p. 209), la langouste géante étouffant un homme (p.212) d'après une fable propagée par OLAus MAGnus, la sabelle (p. 256), la puce de mer, l'« œstre» et les sangsues (p. 268-269), les Hémiptères aquatiques (p. 352) et naturellement le scorpion à élytres du De scorpione.

Il nous faut maintenant conclure:

Le zoologiste aussi bien que l'historien de la Zoologie qui parcourt les in-folios de Gesner ne peut s'empêcher de faire plusieurs constatations.

Et tout d'abord de remarquer à quel point la Zoologie du XVI ${ }^{e}$ siècle est la réplique exacte de celle de l'Antiquité. Tout ce qu'ont dit sur les animaux Aristote, Pline, Elien ou d'autres est parole d'Evangile pour notre savant zürichois.

Bien qu'il ait souvent lui-même fait des observations dans la nature, et notamment dans les montagnes de son pays natal, Gesner est avant tout 
un naturaliste de cabinet et appartient à cette catégorie de savants que nous avons appelée avec G. Petit ${ }^{8}$, celle des érudits polygraphes.

Il ne se contente pas en effet de décrire la morphologie et les mœurs des animaux, mais il faut qu'il ajoute encore à cette description-le plus souvent assez sommaire - des considérations philologiques et philosophiques, des anecdotes et des proverbes, confondant souvent ainsi zoologie et lexicographie.

Ses descriptions ne concernent que l'aspect extérieur des espèces étudiées, l'habitus comme nous dirions aujourd'hui.

La morphologie interne est totalement ignorée et s'il est probable que Gesner avait fait des dissections, celles-ci n'ont pas été utilisées pour la rédaction de son grand ouvrage.

Il faudra attendre le XVII ${ }^{e}$ siècle pour avoir une meilleure connaissance de l'anatomie des animaux, tant Vertébrés qu'Invertébrés. Et pour ce dernier embranchement, c'est Cuvier au XIX ${ }^{\mathbf{e}}$ siècle seulement qui introduira définitivement les critères anatomiques dans la Taxinomie.

Comme nous l'avons déjà fait remarquer avec G. Petit ${ }^{9}$, Gesner a reculé devant les difficultés de la classification zoologique. Pour lui, les termes de genre et d'espèce sont encore fort imprécis, ses « ordres » sont le plus souvent constitués par une réunion d'espèces appartenant en fait à des groupes très différents.

Il a cependant parfois pressenti des affinités naturelles (en classant par exemple l'argonaute avec les autres Céphalopodes) et certaines de ses descriptions sont précises et vivantes. S'il avait vécu plus longtemps, peut-être aurait-il été tenté d'aborder réellement les problèmes de la classification zoologique.

L'iconographie - nous l'avons vu - est souvent intéressante et précise.

Feuilletons par exemple les Icones aquatilium animantium et devant nos yeux défilent, bien reconnaissables, les principaux Invertébrés marins de nos rivages, avec parfois la figuration d'espèces exotiques.

Par contre, le zoologiste d'aujourd'hui sourira devant les invraisemblables figures de monstres marins et devant celle du scorpion ailé.

Gesner est un homme de son temps pour le meilleur et pour le pire. Des gens de la Renaissance qu'ils soient écrivains, artistes ou savants, il a la

8 G. Petit et J.ThÉodonidès, Histoire de la Zoologie, des origines à Linné, Hermann, Paris 1962, p. 254-262.

9 op.cit., p. 261. 
désopilante érudition, le respect sacro-saint des Anciens, mais en même temps il commence à ouvrir ses yeux sur la nature.

Il était cependant trop tôt pour trancher le cordon reliant son siècle à ceux de l'Antiquité et du Moyen-Agé.

Ces restrictions étant faites, on reste confondu d'admiration devant l'immense labeur d'un homme disparu avant la cinquantaine qui a dit lui-même de son opus majus: «J'ai donné tous mes soins à mon ouvrage; je voulais qu'il fut inutile désormais de recourir à d'autres auteurs pour les sujets qu'il traite, ... que l'on eût toute une bibliothèque dans un seul livre.»

Ce but il l'a pleinement atteint lorsque l'on considère l'énorme documentation qu'il nous laisse. Et l'on comprend que Humboldt ${ }^{10}$, cet autre polygraphe ait écrit: «Deux des plus grands hommes du XVI ${ }^{\mathrm{e}}$ siècle, Conrad Gesner et Andreas Cesalpinus ont glorieusement frayé une route nouvelle en zoologie et en botanique.»

10 Kosmos II, p. 335. 